\title{
Desvantagem vocal no canto: análise do protocolo Índice de Desvantagem para o Canto Moderno - IDCM
}

\author{
Voice handicap in singing: analysis of the Modern Singing \\ Handicap Index - MSHI questionnaire
}

\author{
Felipe Moreti ${ }^{1}$, Clara Rocha ${ }^{1}$, Maria Cristina de Menezes Borrego ${ }^{1}$, Mara Behlau ${ }^{1}$
}

\begin{abstract}
RESUMO
Objetivo: Verificar a sensibilidade do protocolo italiano Modern Singing Handicap Index - MSHI, traduzido e culturalmente adaptado para o Português Brasileiro como Índice de Desvantagem para o Canto Moderno - IDCM, comparando os escores de coralistas amadores com e sem queixas vocais e de indivíduos não-cantores, de acordo com gênero, classificação vocal e atividades de canto. Métodos: Duzentos e vinte e seis indivíduos adultos, com idades entre 16 e 66 anos, foram distribuídos em três grupos: 58 cantores com queixas vocais - CCQ; 112 cantores sem queixas vocais - CSQ e 56 indivíduos não cantores e sem queixas vocais - GNC. Os cantores foram selecionados em cinco coros universitários de música popular brasileira, a capella, regidos pelo mesmo maestro. Os indivíduos não cantores foram recrutados nas mesmas instituições dos cantores, com características demográficas semelhantes. Os indivíduos preencheram individualmente o IDCM, questionário com 30 itens divididos em três subescalas: incapacidade (domínio funcional), desvantagem (domínio emocional) e defeito (domínio orgânico). Os cantores também realizaram uma auto-avaliação de suas atividades de canto. Resultados: A média dos escores do IDCM do CCQ $(26,91)$ foi maior que a do o CSQ $(16,61)$, e ambas maiores que a do GNC $(7,79)$. Para os três grupos, a subescala defeito apresentou as maiores médias de escores, seguida por incapacidade e desvantagem. Não houve diferenças dos escores em relação ao gênero, classificação vocal e atividades de canto. Conclusão: O protocolo mostrou-se sensível para cantores modernos com problemas de voz. Coralistas com queixas vocais apresentaram maior desvantagem auto-relatada em relação aos sem queixas e não cantores. Aspectos de natureza orgânica destacaram-se com maiores desvios.
\end{abstract}

Descritores: Voz; Qualidade de vida; Estudos de avaliação; Protocolos; Música; Saúde ocupacional; Questionários

\section{INTRODUÇÃO}

A Organização Mundial da Saúde define saúde como um estado completo de bem-estar físico, mental e social, e não simplesmente como ausência de doença ${ }^{(1)}$. Recentemente, este conceito foi ampliado, incluindo aspectos da qualidade de vida, atualmente definida como a percepção do indivíduo sobre sua posição na vida, em seu contexto cultural e de valores, com relação a seus objetivos, expectativas, padrões e preocupações ${ }^{(1-4)}$. Na avaliação da qualidade de vida, é imprescindível que a percepção do sujeito seja o foco do instrumento de análise. Desta forma, as principais ferramentas para a verificação de consequências variadas de um problema de saúde são questionários para quantificar a avaliação do indivíduo

Trabalho realizado no Centro de Estudos da Voz - CEV - São Paulo (SP), Brasil, como pré-requisito para conclusão do Curso de Especialização em Voz. (1) Centro de Estudos da Voz - CEV - São Paulo (SP), Brasil.

Endereço para correspondência: Felipe Moreti. R. Visconde de Mauá, 347, Vila Assunção, Santo André (SP), Brasil, CEP: 09030-530. E-mail: felipemoreti@uol.com.br

Recebido em: 18/7/2009; Aceito em: 29/10/2009 sobre o impacto de uma alteração em suas relações sociais, profissionais e financeiras ${ }^{(1)}$.

A disfonia representa uma dificuldade ou desvio na produção vocal que, na maioria das vezes, não oferece risco iminente de morte ao indivíduo, sendo seu tratamento geralmente eletivo ${ }^{(5)}$. Por ser um fenômeno multidimensional, a avaliação vocal deve incluir história pregressa da queixa, exame otorrinolaringológico e avaliação fonoaudiológica auditiva e acústica. Porém, a realização destas avaliações não garante a quantificação do problema de voz de acordo com a percepção do indivíduo ${ }^{(6-9)}$.

Pesquisas em quadros vocais severos, como a disfonia espasmódica, reforçam a importância da auto-avaliação durante a avaliação vocal ${ }^{(10)}$, pois nem sempre há relação direta entre a presença de um distúrbio vocal e uma redução na qualidade de vida.

Em casos de uso profissional da voz, a relação entre a presença de um distúrbio vocal e o comprometimento na qualidade de vida parece ser ainda mais complexa, pois em alguns casos como, por exemplo, professores, um desvio vocal pode não limitar sua atividade profissional; enquanto que em outros 
casos, como os cantores, por pertencerem à elite vocal, um pequeno desvio vocal pode causar grande impacto nos aspectos pessoais (físico, mental, social, emocional e comunicativo), profissionais e financeiros ${ }^{(11-13)}$. Os problemas podem ocorrer na voz falada ou serem específicos da voz cantada. Embora todos os problemas na voz do cantor possam ser considerados graves, devido à grande demanda vocal, a percepção deste profissional em relação a sua desvantagem vocal é altamente variável, quer seja pela ampla diversidade do uso da voz em diferentes estilos de canto e horas semanais ou pelo fato de estarem mais atentos às alterações de voz, por usarem a voz como instrumento de trabalho, buscando tratamento adequado muitas vezes no início dos sintomas ${ }^{(14)}$.

Na área de voz, o VHI (Voice Handicap Index) ${ }^{(15)}$ é um dos mais conhecidos e utilizados protocolos de auto-avaliação do impacto de um problema de voz, desenvolvido nos Estados Unidos $^{(16)}$ e validado em quase 20 países $^{(17)}$, inclusive no Brasil, chamado de Índice de Desvantagem Vocal - IDV(18). Usualmente é aplicado em indivíduos adultos com queixas vocais ${ }^{(19)}$ e avalia três diferentes aspectos: incapacidade, desvantagem e defeito vocal.

O termo defeito é definido como qualquer perda ou anormalidade psicológica, fisiológica, anatômica, estrutural, temporária ou permanente. Incapacidade significa qualquer restrição ou diminuição da habilidade de exercer uma atividade habitualmente esperada para o indivíduo. Já a desvantagem é resultante do defeito ou da incapacidade, caracterizada pela limitação ou impedimento no cumprimento de um papel esperado para o indivíduo, ocasionando consequências sociais, culturais, de desenvolvimento e econômicas ${ }^{(20,21)}$.

Apesar da indiscutível validade e confiabilidade do IDV, sua sensibilidade para avaliar cantores é pobre, uma vez que os fatores associados à percepção do indivíduo sobre sua desvantagem na voz cantada não são abordados neste protocolo $^{(22)}$, que não contempla a consequência da disfonia na vida de cantores ${ }^{(14,23,24)}$.

Para atender a essa população, foram desenvolvidas adaptações do IDV para a voz cantada ${ }^{(24,25)}$. O foniatra italiano Franco Fussi propôs duas versões, após análise de mais de 400 cantores: o Modern Singing Handicap Index - MSHI (Índice de Desvantagem para o Canto Moderno - IDCM) e o Classical Singing Handicap Index - CSHI (Índice de Desvantagem para o Canto Clássico - IDCC) $)^{(26)}$.

O objetivo do presente estudo é verificar a sensibilidade do protocolo italiano Modern Singing Handicap Index - MSHI, traduzido e culturalmente adaptado para o Português Brasileiro como Índice de Desvantagem do Canto Moderno - IDCM, comparando os escores de coralistas amadores de canto moderno, com e sem queixas de voz, com os de indivíduos não-cantores, de acordo com gênero, classificação vocal e atividades de canto.

\section{MÉTODOS}

O estudo foi aprovado pelo Comitê de Ética em Pesquisa do Centro de Estudo da Voz (CEP-CEV/ISEC 1215/07). Todos os sujeitos envolvidos (ou seus responsáveis) assinaram do Termo de Consentimento Livre e Esclarecido, consentindo, desta forma, com a realização e divulgação desta pesquisa e de seus resultados conforme Resolução 196/96 (BRASIL. Resolução MS/CNS/CNEP nº 196/96 de 10 de outubro de 1996).

Participaram 226 voluntários entre 16 e 66 anos de idade, distribuídos em três grupos: 58 cantores com queixas vocais - CCQ, 112 cantores sem queixas vocais - CSQ e 56 indivíduos não cantores e sem queixas vocais, o grupo não cantor - GNC. Os cantores eram homens (32 tenores e 48 baixos) e mulheres (49 sopranos e 41 contraltos), pertencentes a cinco coros universitários de música popular brasileira, a capella, regidos pelo mesmo maestro. Eles deveriam, obrigatoriamente, pertencer ao coro há pelo menos seis meses, com média de cinco horas de ensaio por semana, em dois dias alternados. Todos realizam aquecimento vocal com tempo variando entre 20 e 30 minutos. Os indivíduos do GNC foram recrutados nas mesmas instituições dos cantores, com características demográficas semelhantes.

Todos os coralistas amadores preencheram um questionário de auto-avaliação, contendo dados de identificação, gênero, data de nascimento, nome do coro, classificação vocal, tempo de canto, tempo de canto em coros, tempo no coro atual, horas de aula de canto e/ou técnica vocal por semana e horas semanais de ensaio do coro, presença de problemas vocais (não, sim, às vezes), sendo que em caso positivo (sim e às vezes) o indivíduo foi orientado a escrever desde quando e se há presença de sintomas na garganta (ardor, coceira, dor, sensação de secura, queimação, sensação de aperto ou bola).

O protocolo MSHI foi traduzido e culturalmente adaptado para o Português Brasileiro como Índice de Desvantagem para o Canto Moderno - IDCM ${ }^{(27)}$ (Anexo 1) e aplicado individualmente. O IDCM é composto por 30 itens, distribuídos em três subescalas: incapacidade, desvantagem e defeito, que correspondem respectivamente aos domínios funcional (exemplo: "Por causa do meu problema de voz sou forçado a limitar meu tempo de estudo/ensaio"), emocional (exemplo: "Fico preocupado quando me pedem para repetir um vocalize ou uma frase musical") e orgânico (exemplo: "Tenho problemas com o controle da respiração para o canto"). As respostas foram assinaladas em uma escala de Likert de cinco pontos de acordo com a frequência de ocorrência: 0: nunca, 1: quase nunca, 2: às vezes, 3: quase sempre e 4: sempre. O IDCM apresenta quatro escores: incapacidade (funcional), desvantagem (emocional) e defeito (orgânico), cada um com valor máximo de 40 pontos e o total, composto pela somatória dos anteriores, com um desvio máximo de 120 pontos. Quanto maior a pontuação, maior a desvantagem percebida pelo indivíduo.

Os dados foram tabulados e analisados da seguinte forma: comparação das médias dos escores do IDCM entre os gêneros dos três grupos; comparação das médias dos escores do IDCM entre os naipes dos grupos de cantores com e sem queixas vocais; comparação das médias dos escores das três subescalas e total do IDCM dos três grupos e levantamento dos dados do questionário de auto-avaliação.

Para a análise estatística, adotou-se o nível de significância de $5 \%(0,05)$. Foram utilizados os testes não paramétricos de Mann-Whitney, Kruskal-Wallis, Friedman e Wilcoxon. Na complementação da análise descritiva, utilizou-se a técnica de Intervalo de Confiança para Média. 
Tabela 1. Médias dos escores das subescalas e totais do IDCM por gênero

\begin{tabular}{|c|c|c|c|c|c|c|c|c|c|}
\hline \multirow{2}{*}{ Gênero } & & \multicolumn{2}{|c|}{ Incapacidade } & \multicolumn{2}{|c|}{ Desvantagem } & \multicolumn{2}{|c|}{ Defeito } & \multicolumn{2}{|c|}{ Total } \\
\hline & & $\mathrm{F}$ & $\mathrm{M}$ & $\mathrm{F}$ & $\mathrm{M}$ & $\mathrm{F}$ & $\mathrm{M}$ & $\mathrm{F}$ & $\mathrm{M}$ \\
\hline & Média & 8,45 & 6,80 & 4,63 & 5,85 & 13,74 & 14,45 & 26,82 & 27,10 \\
\hline \multirow[t]{3}{*}{ Grupo CCQ } & $\mathrm{n}$ & 38 & 20 & 38 & 20 & 38 & 20 & 38 & 20 \\
\hline & Valor de $p$ & \multicolumn{2}{|c|}{0,329} & \multicolumn{2}{|c|}{0,993} & \multicolumn{2}{|c|}{0,838} & \multicolumn{2}{|c|}{0,928} \\
\hline & Média & 4,96 & 4,40 & 2,85 & 3,43 & 8,73 & 8,83 & 16,54 & 16,67 \\
\hline \multirow{3}{*}{ Grupo CSQ } & $\mathrm{n}$ & 52 & 60 & 52 & 60 & 52 & 60 & 52 & 60 \\
\hline & Valor de $p$ & \multicolumn{2}{|c|}{0,740} & \multicolumn{2}{|c|}{0,440} & \multicolumn{2}{|c|}{0,875} & \multicolumn{2}{|c|}{0,829} \\
\hline & Média & 2,50 & 1,96 & 1,43 & 1,68 & 3,54 & 4,39 & 7,54 & 8,04 \\
\hline \multirow[t]{2}{*}{ Grupo GNC } & $\mathrm{n}$ & 28 & 28 & 28 & 28 & 28 & 28 & 28 & 28 \\
\hline & Valor de $p$ & \multicolumn{2}{|c|}{0,532} & \multicolumn{2}{|c|}{0,643} & \multicolumn{2}{|c|}{0,731} & \multicolumn{2}{|c|}{0,928} \\
\hline
\end{tabular}

Teste Mann-Whitney $(p \leq 0,05)$

Legenda: $\mathrm{F}$ = feminino; $\mathrm{M}$ = masculino; Grupo $\mathrm{CCQ}$ = grupo cantores com queixas; Grupo $\mathrm{CSQ}$ = grupo cantores sem queixas; Grupo GNC = grupo não cantores

\section{RESULTADOS}

Os resultados do IDCM indicaram que as médias dos escores das subescalas (incapacidade, desvantagem e defeito)

Tabela 2. Médias dos escores das subescalas e totais do IDCM por naipe

\begin{tabular}{|c|c|c|c|c|c|}
\hline & $\begin{array}{l}\text { Subescalas do } \\
\text { IDCM }\end{array}$ & Naipe & $\begin{array}{c}\text { Média do } \\
\text { escore }\end{array}$ & $\mathrm{n}$ & Valor de $p$ \\
\hline \multirow{16}{*}{$\begin{array}{l}0 \\
0 \\
0 \\
\circ \\
\circ \\
\end{array}$} & \multirow{4}{*}{ Incapacidade } & Baixo & 5,50 & 10 & \multirow{4}{*}{0,335} \\
\hline & & Contralto & 9,71 & 17 & \\
\hline & & Soprano & 7,43 & 21 & \\
\hline & & Tenor & 8,10 & 10 & \\
\hline & \multirow{4}{*}{ Desvantagem } & Baixo & 4,20 & 10 & \multirow{4}{*}{0,091} \\
\hline & & Contralto & 6,41 & 17 & \\
\hline & & Soprano & 3,19 & 21 & \\
\hline & & Tenor & 7,50 & 10 & \\
\hline & \multirow{4}{*}{ Defeito } & Baixo & 15,80 & 10 & \multirow{4}{*}{0,418} \\
\hline & & Contralto & 16,06 & 17 & \\
\hline & & Soprano & 11,86 & 21 & \\
\hline & & Tenor & 13,10 & 10 & \\
\hline & \multirow{4}{*}{ Total } & Baixo & 25,50 & 10 & \multirow{4}{*}{0,447} \\
\hline & & Contralto & 32,18 & 17 & \\
\hline & & Soprano & 22,48 & 21 & \\
\hline & & Tenor & 28,70 & 10 & \\
\hline \multirow{16}{*}{ 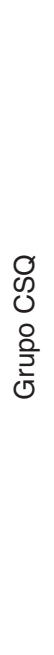 } & \multirow{4}{*}{ Incapacidade } & Baixo & 4,68 & 38 & \multirow{4}{*}{0,718} \\
\hline & & Contralto & 4,50 & 24 & \\
\hline & & Soprano & 5,36 & 28 & \\
\hline & & Tenor & 3,91 & 22 & \\
\hline & \multirow{4}{*}{ Desvantagem } & Baixo & 3,16 & 38 & \multirow{4}{*}{0,579} \\
\hline & & Contralto & 3,21 & 24 & \\
\hline & & Soprano & 2,54 & 28 & \\
\hline & & Tenor & 3,91 & 22 & \\
\hline & \multirow{4}{*}{ Defeito } & Baixo & 8,74 & 38 & \multirow{4}{*}{0,953} \\
\hline & & Contralto & 8,88 & 24 & \\
\hline & & Soprano & 8,61 & 28 & \\
\hline & & Tenor & 9,00 & 22 & \\
\hline & \multirow{4}{*}{ Total } & Baixo & 16,58 & 38 & \multirow{4}{*}{0,983} \\
\hline & & Contralto & 16,58 & 24 & \\
\hline & & Soprano & 16,50 & 28 & \\
\hline & & Tenor & 16,82 & 22 & \\
\hline
\end{tabular}

Teste Kruskal-Wallis $(p \leq 0,05)$

Legenda: Grupo $\mathrm{CCQ}=$ grupo cantores com queixas; Grupo CSQ = grupo cantores sem queixas e total foram semelhantes entre os gêneros (feminino e masculino) e entre os naipes (baixo, contralto, soprano e tenor) (Tabelas 1 e 2).

Comparando as médias dos escores dos grupos CCQ, CSQ e GNC nas três subescalas do IDCM, incapacidade, desvantagem e defeito, observou-se diferenças significativas em todas as comparações, sendo a subescala defeito a que apresentou as maiores médias de escores, seguida pelas subescalas incapacidade e desvantagem (Tabela 3 ).

Tabela 3. Comparação das médias dos escores das subescalas do IDCM

\begin{tabular}{|c|c|c|c|c|}
\hline \multicolumn{2}{|c|}{ Subescalas } & \multirow{2}{*}{$\begin{array}{c}\text { Média } \\
7,88\end{array}$} & \multirow{2}{*}{$\frac{n}{58}$} & \multirow[t]{2}{*}{ Valor de $p$} \\
\hline & Incapacidade & & & \\
\hline \multirow[t]{3}{*}{ Grupo CCQ } & Desvantagem & 5,05 & 58 & $<0,001^{*}$ \\
\hline & Defeito & 13,98 & 58 & \\
\hline & Incapacidade & 4,66 & 112 & \\
\hline \multirow[t]{3}{*}{ Grupo CSQ } & Desvantagem & 3,16 & 112 & $<0,001^{*}$ \\
\hline & Defeito & 8,79 & 112 & \\
\hline & Incapacidade & 2,23 & 56 & \\
\hline \multirow[t]{2}{*}{ Grupo GNC } & Desvantagem & 1,55 & 56 & $<0,001^{*}$ \\
\hline & Defeito & 3,96 & 56 & \\
\hline
\end{tabular}

*Valores significativos $(p \leq 0,05)$ - Teste Friedman

Legenda: Grupo $\mathrm{CCQ}$ = grupo cantores com queixas; Grupo $\mathrm{CSQ}=$ grupo cantores sem queixas; Grupo GNC = grupo não cantores

Comparou-se as médias dos escores dos três grupos em todas as subescalas e no total. Verificou-se que existe diferença significativa entre os grupos para todas as comparações (Tabela 4), sendo que o grupo CCQ teve sempre os maiores resultados e o GNC os menores.

Para finalizar, comparou-se as médias dos escores dos grupos CCQ e CSQ nos itens do questionário de auto-avaliação. Verificou-se que não existem diferenças significativas entre os grupos em nenhuma das respostas do questionário (Tabela 5).

\section{DISCUSSÃO}

Existe informação limitada a respeito dos problemas que afetam a qualidade de vida do profissional da voz, ainda mais no caso de cantores amadores. O que se sabe atualmente é que os distúrbios vocais nestes profissionais resultam em mudan- 
Tabela 4. Comparação dos três grupos em cada subescala

\begin{tabular}{lcccc}
\hline \multicolumn{1}{c}{ Grupo } & & Média & $\mathrm{n}$ & Valor de $\mathrm{p}$ \\
\hline \multirow{4}{*}{ Incapacidade } & $\mathrm{CCQ}$ & 7,88 & 58 & \\
& $\mathrm{CSQ}$ & 4,66 & 112 & $<0,001^{*}$ \\
& $\mathrm{GNC}$ & 2,23 & 56 & \\
\hline \multirow{4}{*}{ Desvantagem } & $\mathrm{CCQ}$ & 5,05 & 58 & \\
& $\mathrm{CSQ}$ & 3,16 & 112 & $<0,001^{*}$ \\
& $\mathrm{GNC}$ & 1,55 & 56 & \\
\hline \multirow{3}{*}{ Defeito } & $\mathrm{CCQ}$ & 13,98 & 58 & \\
& $\mathrm{CSQ}$ & 8,79 & 112 & $<0,001^{*}$ \\
& $\mathrm{GNC}$ & 3,96 & 56 & \\
Total & $\mathrm{CCQ}$ & 26,91 & 58 & \\
& $\mathrm{CSQ}$ & 16,61 & 112 & $<0,001^{*}$ \\
& $\mathrm{GNC}$ & 7,79 & 56 & \\
\hline
\end{tabular}

* Valores significativos $(p \leq 0,05)$ - Teste Kruskal-Wallis

Legenda: Grupo $\mathrm{CCQ}$ = grupo cantores com queixas; Grupo $\mathrm{CSQ}=$ grupo cantores sem queixas; Grupo GNC = grupo não cantores

Tabela 5. Questionário de auto-avaliação

\begin{tabular}{lccc}
\hline Questionário & Grupo & Média & Valor de p \\
\hline Tempo no coro atual (anos) & CCQ & 2,28 & \multirow{2}{*}{0,832} \\
Tempo de canto em coros & CCQ & 2,18 & \\
(anos) & CSQ & 5,53 & 0,826 \\
Tempo de canto (anos) & CCQ & 7,73 & \\
& CSQ & 6,80 & 0,456 \\
Aula de canto (horas/semana) & $\mathrm{CCQ}$ & 0,74 & \\
& $\mathrm{CSQ}$ & 1,12 & 0,176 \\
Técnica vocal (horas/semana) & $\mathrm{CCQ}$ & 0,73 & \\
& $\mathrm{CSQ}$ & 0,96 & 0,240 \\
Horas de ensaio por semana & $\mathrm{CCQ}$ & 4,78 & \\
& $\mathrm{CSQ}$ & 5,37 & 0,201 \\
\hline
\end{tabular}

Teste Mann-Whitney $(p \leq 0,05)$

Legenda: Grupo $\mathrm{CCQ}$ = grupo cantores com queixas; Grupo $\mathrm{CSQ}=$ grupo cantores sem queixas

ças, adaptações e/ou interrupções na rotina diária. Além disto, o estilo de vida, o ambiente social e o local onde se utiliza profissionalmente a voz podem contribuir para o surgimento ou manutenção dos distúrbios vocais ${ }^{(11,12,28)}$. O impacto de uma disfonia em profissionais da voz geralmente é muito grave e pode comprometer definitivamente a carreira ${ }^{(11)}$ ou limitar seus hobbies ou atividades de lazer.

Embora se saiba que os distúrbios vocais podem causar impactos importantes nas atividades diárias e na qualidade de vida das pessoas, existem poucos instrumentos que quantifiquem adequadamente o impacto destes distúrbios na vida dos indivíduos ${ }^{(14,28)}$, e mais especificamente em profissionais da voz.

Os coralistas amadores homens e mulheres, de diferentes naipes, apresentam dificuldades semelhantes, o que confirma a não interferência desses aspectos do canto, por fatores que podem envolver cuidado na classificação vocal pelo regente, distribuição homogênea nos diversos naipes e semelhanças na exigência vocal ${ }^{(14,28)}$.

Os valores do IDCM foram claramente maiores para os cantores, mostrando a relevância de se ter um protocolo sensível à população na qual é aplicado ${ }^{(29)}$. Na comparação entre as três subescalas, o defeito, que corresponde ao domínio orgâ- nico, apresentou os maiores escores, seguido pelas subescalas incapacidade e desvantagem, que representam respectivamente os domínios funcional e emocional. Fatores como falta de domínio técnico, demanda vocal elevada na fala e limitada experiência no canto podem explicar esses resultados ${ }^{(28)} \mathrm{e}$, consequentemente, colocar esses indivíduos em uma situação de risco vocal potencial ${ }^{(24)}$. Os valores reduzidos do domínio emocional podem indicar que os coralistas amadores não dependem das atividades de canto para sobreviver e, assim, um pequeno desvio na qualidade vocal pode ser aceito sem produzir consequências psicológicas, o que não ocorre com o cantor profissional, em que, pelo fato de depender financeiramente de sua voz, qualquer alteração mínima na qualidade vocal gera grande impacto em sua qualidade de vida ${ }^{(14)}$, principalmente na esfera psicológica ${ }^{(29)}$.

A comparação dos escores do IDCM dos grupos CCQ, CSQ e GNC e também dos grupos entre si mostrou-se significativa em todas as análises, sendo que a presença de queixa vocal foi determinante na manifestação do desvio. Considerando que o protocolo IDCM quantifica a desvantagem vocal nas atividades de canto causada por um problema de voz de qualquer natureza, quanto maior a presença de queixas vocais no canto, maior a probabilidade dos escores do IDCM estarem aumentados, o que mostra a sensibilidade do protocolo para a população de cantores com queixas ${ }^{(14,28)}$.

Por fim, as comparações dos grupos CCQ e CSQ, em relação ao questionário de auto-avaliação das atividades de canto, mostraram que queixas vocais presentes no grupo CCQ não podem ser explicadas por diferenças na rotina de uso da voz cantada, visto que ambos os grupos se comportam de maneira semelhante.

$\mathrm{O}$ uso incorreto da voz pelo cantor pode prejudicar sua saúde vocal. Assim, a compreensão sobre as dificuldades e queixas vocais de coralistas poderá auxiliar fonoaudiólogos, preparadores vocais e regentes a proporcionar condições saudáveis de uso da voz cantada a essa população.

\section{CONCLUSÃO}

Cantores modernos com queixas vocais apresentaram maior desvantagem auto-relatada em relação aos sem problemas de voz e não cantores. No relato dos cantores modernos com problemas, aspectos identificados como sendo de natureza orgânica destacaram-se com maiores desvios, refletindo o tipo de dificuldade desses indivíduos. O protocolo mostrouse sensível para essa população, podendo ser utilizado para auxiliar fonoaudiólogos, preparadores vocais e regentes no mapeamento de problemas vocais.

\section{AGRADECIMENTOS}

Agradecemos ao Maestro Eduardo Gonçalves Fernandes, regente dos coros participantes desta pesquisa (Coral UNIFESP, Coralusp XI de Agosto, Coral Belas Artes, Coral FEA-USP e Coral da FAAM) pelo carinho, acolhimento, incentivo, apoio incondicional e a viabilidade da coleta da amostra durante os ensaios dos coros. 


\begin{abstract}
Purpose: To assess the sensitivity of the Italian self-assessment questionnaire Modern Singing Handicap Index - MSHI, translated and culturally adapted to Brazilian Portuguese as Índice de Desvantagem para o Canto Moderno - IDCM, comparing scores of amateur choir singers with or without voice complaints and non-singers according to gender, singing voice classification and singing activities. Methods: Two hundred twenty-six adults with ages between 16 and 66 years were divided into three groups: 58 singers with vocal complaints - SC; 112 singers without vocal complaints - SwC and 56 non-singers without vocal complaints - NS. The singers were selected from five university choirs of a capella Brazilian popular music, lead by the same conductor. The non-singers were recruited at the same institutions of the singers with similar demographic characteristics. The subjects filled the IDCM individually. The IDCM is a questionnaire with 30 items divided into three subscales: disability (functional domain), handicap (emotional domain) and impairment (organic domain). The singers also did a self-assessment of their singing activities. Results: The mean IDCM score of the SC group (26.91) was higher than the that of the SwC (16.61), and both were higher than that of the NS group (7.79). For the three groups, the impairment subscale showed higher scores, followed by disability and handicap. There were no score differences regarding gender, singing voice classification and singing activities. Conclusion: The questionnaire proved to be sensitive for modern singers with vocal complaints. Choir singers with vocal complaints had higher self-reported handicap in comparison to choir singers without vocal complaints and non-singers. Aspects of organic nature were highlighted with larger deviations.
\end{abstract}

Keywords: Voice; Quality of life; Evaluation studies; Protocols; Music; Occupational health; Questionnaires

\title{
REFERENCIAS
}

1. World Health Organization. WHOQOL. Measuring Quality of Life. The World Health Organization Quality of Life Instruments. (THE WHOQOL-100 AND THE WHOQOL-BREF). WHO/MSA/MNH/ PSF/97.4. 1997. p.1-15.

2. Guyatt GH, Feeny DH, Patrick DL. Measuring health-related quality of life. Ann Intern Med. 1993;118(8):622-9.

3. Gill TM, Feinstein AR. A critical appraisal of the quality of quality-oflife measurements. JAMA. 1994;272(8):619-26.

4. Barbotte E, Guillemin F, Chau N; Lorhandicap Group. Prevalence of impairments, disabilities, hadicaps and quality of life in the general population: a review of recent literature. Bull World Health Organ. 2001;79(11):1047-55.

5. Behlau M, Madazio G, Feijó D, Pontes P. Avaliação da voz. In: Behlau M, organizadora. Voz: o livro do especialista. Rio de Janeiro: Revinter; 2001. V.1, p. 83-245.

6. Gasparini G, Behlau M. Quality of life: validation of the Brazilian version of the voice-related quality of life (V-RQOL) measure. J Voice. 2009;23(1):76-81.

7. Behlau M, Hogikyan ND, Gasparini G. Quality of life and voice: study of a Brazilian population using the voice-related quality of life measure. Folia Phoniatr Logop. 2007;59(6):286-96.

8. Rosen CA, Murry T, Zinn A, Zullo T, Sonbolian M. Voice handicap index change following treatment of voice disorders. J Voice. 2000;14(4):619-23.

9. Señaris Gonzáles B, Nuñez Batalla F, Corte Santos P, Suárez Nieto C. Indice de Incapacidad Vocal: factores predictivos. Acta Otorrinolaringol Esp. 2006;57(2):101-8.

10. Hogikyan ND, Wodchis WP, Spak C, Kileny PR. Longitudinal effects of botulinum toxin injections on voice-related quality of life (V-RQOL) for patients with adductory spasmodic dysphonia. J Voice. 2001;15(4):57686.

11. Sataloff RT. Voice impairment, disability, handicap, and medical-legal evaluation. In: Sataloff RT, editor. Professional voice: the science and the art of clinical care. 2nd ed. San Diego: Singular; 2005. p.1433-41.

12. Wingate JM, Brown WS, Shrivastav R, Davenport P, Sapienza CM. Treatment outcomes for professional voice users. J Voice. 2007;21(4):433-49.

13. Franic DM, Bramlett RE, Bothe AC. Psychometric evaluation of disease specific quality of life instruments in voice disorders. J Voice. 2005;19(2):300-15.

14. Rosen CA, Murry T. Voice handicap index in singers. J Voice. 2000;14(3):370-7.

15. Grässel E, Hoppe U, Rosanowski F. [Grading of the Voice Handicap Index]. HNO. 2008;56(12):1221-8. German.
16. Jacobson BH, Johnson A, Grywalski C, Silbergleit A, Jacobson G, Benninger MS, Newman CW. The Voice Handicap Index (VHI): development and validation. Am J Speech Lang Pathol. 1997;6:66-70.

17. Verdonck-de Leeuw IM, Kuik DJ, De Bodt M, Guimaraes I, Holmberg EB, Nawka T, et al. Validation of the voice handicap index by assessing equivalence of European translations. Folia Phoniatr Logop. 2008;60(4):173-8.

18. Behlau M, Alves dos Santos L de M, Oliveira G. Cross-cultural adaptation and validation of the voice handicap index into brazilian portuguese. J Voice. 2011;25(3):354-9.

19. Zur KB, Cotton S, Kelchner L, Baker S, Weinrich B, Lee L. Pediatric Voice Handicap Index (pVHI): a new tool for evaluating pediatric dysphonia. Int J Pediatr Otorhinolaryngol. 2007;71(1):77-82.

20. World Health Organization. International Classification of Functioning, Disability and Health. Geneva: WHO; 2001.

21. Cocchiarella L, Andersson GBJ, editors. Guides to the Evaluation of Permanent Impairment. 5th ed. Chicago: American Medical Association; 2001.

22. Cohen SM, Noordzij JP, Garrett CG, Ossoff RH. Factors associated with perception of singing voice handicap. Otolaryngol Head Neck Surg. 2008;138(4):430-4.

23. Behrman A, Sulica L, He T. Factors predicting patient perception of dysphonia caused by benign vocal fold lesions. Laryngoscope. 2004;114(10):1693-700.

24. Cohen SM, Jacobson BH, Garrett CG, Noordzij JP, Stewart MG, Attia A, et al. Creation and validation of the Singing Voice Handicap Index. Ann Otol Rhinol Laryngol. 2007;116(6):402-6.

25. Morsomme D, Gaspar M, Jamart J, Remacle M, Verduyckt I. Adaptation du Voice Handicap Index à la voix chantée. Rev Laryngol Otol Rhinol. 2007;128(5):305-14.

26. Fussi F, Fuschini T. Foniatria artistica: la presa in carico foniatricologopedica del cantante classico e moderno. Audiol Foniatr. 2008;13(12):4-28.

27. Moreti F, Silva C, Borrego MC, Behlau M. Desvantagem vocal no canto: análise do protocolo IDCM [Internet]. In: $17^{\circ}$ Congresso Brasileiro de Fonoaudiologia; 2009 Out 21-24; Salvador. Anais eletrônicos. [citado 2011 Maio 9]. Disponível em: www.sbfa.org.br/portal/anais2009/ resumos/R1480-1.pdf.

28. Jotz GP, Bramati O, Schimidt VB, Dornelles S, Gigante LP. Aplicação do "Voice Handicap Index" em coralistas. Arq Otorrinolaringol. 2002;6(4):260-4.

29. Murry T, Zschommler A, Prokop J. Voice handicap in singers. J Voice. 2009;23(3):376-9. 
Anexo 1. Versão brasileira do protocolo Modern Singing Handicap Index - MSHI, chamado Índice de Desvantagem para o Canto Moderno - IDCM ${ }^{(27)}$

Marque a resposta que indica o quanto você compartilha da mesma experiência:

Chave de resposta: 0: nunca; 1: quase nunca; 2 : às vezes; 3: quase sempre; 4: sempre

O impacto do problema de voz nas atividades profissionais

Disability - Incapacidade

\begin{tabular}{|l|l|l|l|l|l|l|}
\hline 1 & Sinto minha voz cansada desde o começo de uma apresentação. & 0 & 1 & 2 & 3 & 4 \\
\hline 2 & Minha voz fica cansada ou alterada durante a apresentação. & 0 & 1 & 2 & 3 & 4 \\
\hline 3 & Tenho que ajustar a minha técnica vocal, porque o problema de voz prejudica a minha emissão. & 0 & 1 & 2 & 3 & 4 \\
\hline 4 & $\begin{array}{l}\text { Meu problema vocal me obriga a modificar as músicas, limitar meu repertório ou mesmo mudar } \\
\text { o tom. }\end{array}$ & 0 & 1 & 2 & 3 & 4 \\
\hline 5 & Por causa do meu problema de voz sou forçado a limitar meu tempo de estudo/ensaio. & 0 & 1 & 2 & 3 & 4 \\
\hline 6 & Sinto dificuldade nas apresentações por causa das alterações no meu rendimento vocal. & 0 & 1 & 2 & 3 & 4 \\
\hline 7 & Não consigo fazer duas ou mais apresentações consecutivas. & 0 & 1 & 2 & 3 & 4 \\
\hline 8 & Preciso da ajuda do operador de som para mascarar meu problema de voz. & 0 & 1 & 2 & 3 & 4 \\
\hline 9 & Preciso tomar remédios continuamente para mascarar meu problema de voz. & 0 & 1 & 2 & 3 & 4 \\
\hline 10 & Meu problema vocal me obriga a limitar o uso social da voz. & 0 & 1 & 2 & 3 & 4 \\
\hline
\end{tabular}

O impacto psicológico do problema de voz

Handicap - Desvantagem

\begin{tabular}{|l|l|l|l|l|l|l|}
\hline 1 & Minha ansiedade antes das apresentações está maior que a habitual. & 0 & 1 & 2 & 3 & 4 \\
\hline 2 & As pessoas com as quais convivo não compreendem minha queixa de voz. & 0 & 1 & 2 & 3 & 4 \\
\hline 3 & As pessoas com as quais convivo têm criticado a minha voz. & 0 & 1 & 2 & 3 & 4 \\
\hline 4 & Meu problema de voz me deixa nervoso e/ou menos sociável. & 0 & 1 & 2 & 3 & 4 \\
\hline 5 & Fico preocupado quando me pedem para repetir um vocalize ou uma frase musical. & 0 & 1 & 2 & 3 & 4 \\
\hline 6 & Sinto que minha carreira está em risco por causa do meu problema de voz. & 0 & 1 & 2 & 3 & 4 \\
\hline 7 & Colegas, empresários e críticos já perceberam minhas dificuldades vocais. & 0 & 1 & 2 & 3 & 4 \\
\hline 8 & Sou obrigado a cancelar alguns compromissos profissionais por causa da voz. & 0 & 1 & 2 & 3 & 4 \\
\hline 9 & Evito agendar futuros compromissos profissionais. & 0 & 1 & 2 & 3 & 4 \\
\hline 10 & Evito conversar com as pessoas. & 0 & 1 & 2 & 3 & 4 \\
\hline
\end{tabular}

Auto-percepção das características de minha voz Impairment - Defeito

\begin{tabular}{|l|l|l|l|l|l|l|}
\hline 1 & Tenho problemas com o controle da respiração para o canto. & 0 & 1 & 2 & 3 & 4 \\
\hline 2 & Meu rendimento vocal varia durante o dia. & 0 & 1 & 2 & 3 & 4 \\
\hline 3 & Sinto que minha voz está fraca ou tem ar na voz. & 0 & 1 & 2 & 3 & 4 \\
\hline 4 & Sinto minha voz rouca. & 0 & 1 & 2 & 3 & 4 \\
\hline 5 & Sinto que tenho que forçar minha voz para produzir os sons. & 0 & 1 & 2 & 3 & 4 \\
\hline 6 & Meu rendimento vocal varia de modo imprevisível durante as apresentações. & 0 & 1 & 2 & 3 & 4 \\
\hline 7 & Tento modificar minha voz para melhorar a qualidade. & 0 & 1 & 2 & 3 & 4 \\
\hline 8 & Cantar está sendo uma tarefa difícil ou cansativa. & 0 & 1 & 2 & 3 & 4 \\
\hline 9 & Minha voz fica pior à noite. & 0 & 1 & 2 & 3 & 4 \\
\hline 10 & Minha voz fica facilmente cansada durante as apresentações. & 0 & 1 & 2 & 3 & 4 \\
\hline
\end{tabular}

